\title{
Observations of the Antonyms of Nouns and Adjectives in the Economic Terminology in the Albanian Language
}

\author{
Sadete Pllana \\ Prof. Ass. Dr. , University of Prishtina "Hasan Prishtina" Prishtina \\ Faculty of Economics Prishtina Republic of Kosova \\ Albulena Pllana Breznica \\ Technical High School "Lutfi Musiqi” Vushtrri Republic of Kosova \\ Fisnike Pllana \\ University of Prishtina "Hasan Prishtina" Prishtina \\ Faculty of Philology Prishtina Republic of Kosova
}

\begin{abstract}
The antonymy arouses various discussions in various studies, especially the extreme or scalable opposition, it is complete and partial, negation and opposition. On the basis of many studies it is argued and accepted that the antonyms deny each other but at the same time assert themselves.Compound antonymic words are more numerous than the merged words (agglutinations). By analyzing the syntactic relationships between the elements of these words, they emerge entirely only as deterministic compounds. From the lexical-grammatical point of view these are nouns, adjectives and few adverbs and pronouns. In this work, the antonymic pairs, nouns and adjectives, are treated, which are widely used in the Albanian economic terminology. The systemic organization of terminology is also helped by the antonymic contrasts. Contrary to synonymy and homonymy, the antonymy characterizes the terminology positively, precisely because it proves the systematic connection of terms for a developed terminology in the system.
\end{abstract}

Keywords: antonyms, economic terminology, antonymic noun pairs, antonymic adjective pairs.

\section{Introduction}

Antonyms are of great interest to lexicography. They play an important role in fulfilling the meaning of words through opposition. Consciously, the antonyms were first listed in the Dictionary of Kristoforidhi. He has made a great contribution in this regard, by reflecting hundreds of antonyms. A pretty good work on the reflection of antonyms has also been done later in the Dictionary of the Albanian Contemporary Language $(1980)^{2}$.

The antonymy appears as a linguistic phenomenon with a systemic character, both for the general language and for the terminology, as they create connections from the opposite; so it is a phenomenon of linking meanings or concepts that are in opposition to each other. In regard to terminology, this phenomenon can occur to varying degrees depending on the nature of the field. This phenomenon is also reflected in the terminology of the economy, especially in its basic vocabulary. As a rule, adjective units can be opposed because they show qualities, and fewer noun units because they mark concrete objects:

Adjective units: i përshpejtuar - i ngadalësuar (accelerated — decelerated); homogjen — heterogjen (homogeneous — heterogeneous); etc.

Noun units: mikrostrukturë - makrostrukturë (microstructure - macrostructure); mikroorganizim (micro-organization) makroorganizim (macro-organization); etc. 
Antonymic relationships are also the most important manifestations of the systemic nature of language.

Among the determining antonymic compounds of nouns, the noun + noun type is the most common:

\begin{tabular}{|c|c|c|c|c|c|}
\hline Albanian & noun + noun & English & Albanian & noun + noun & English \\
\hline granddhënes & $\begin{array}{l}\text { grand + dhënes } \\
\text { grant + giver }\end{array}$ & grantor & grandmarrës & $\begin{array}{l}\text { grand + marrës } \\
\text { grant + taker }\end{array}$ & grantee \\
\hline huadhënës & $\begin{array}{l}\text { hua + dhënes } \\
\text { loan + giver }\end{array}$ & lender & huamarrës & $\begin{array}{l}\text { hua + marrës } \\
\text { loan + taker }\end{array}$ & borrower \\
\hline qiradhënie & $\begin{array}{l}\text { qira + dhënie } \\
\text { rent + giving }\end{array}$ & leasing & qiramarrje & $\begin{array}{l}\text { qira + marrje } \\
\text { rent + taking }\end{array}$ & hiring \\
\hline qiradhënës & $\begin{array}{l}\text { qira + dhënës } \\
\text { rent + giver }\end{array}$ & $\begin{array}{l}\text { landlord, } \\
\text { lessor }\end{array}$ & qiramarrës & $\begin{array}{l}\text { qira + marrës } \\
\text { rent + taker }\end{array}$ & tenant, lessee \\
\hline pikënisje & $\begin{array}{l}\text { pikë + nisje } \\
\text { point + start }\end{array}$ & $\begin{array}{l}\text { starting } \\
\text { point }\end{array}$ & pikëarritje & $\begin{array}{l}\text { pike + arritje } \\
\text { point + arrival }\end{array}$ & arrival point \\
\hline
\end{tabular}

Some determining antonymic compounds of nouns, of the adverb + noun type:

\begin{tabular}{|l|l|l|l|l|l|}
\hline Albanian & adverb + noun & English & Albanian & adverb + noun & English \\
\cline { 2 - 5 } & $\begin{array}{l}\text { mirë + besim } \\
\text { good + faith }\end{array}$ & good faith & $\begin{array}{l}\text { keq + besim } \\
\text { bad + faith }\end{array}$ & bad faith \\
\cline { 2 - 5 } & $\begin{array}{l}\text { mirë + kuptim } \\
\text { good + } \\
\text { understäkuptim }\end{array}$ & understanding & $\begin{array}{l}\text { keq + kuptim } \\
\text { bad + understanding }\end{array}$ & $\begin{array}{l}\text { misunder- } \\
\text { standing }\end{array}$ \\
\hline mirëqeverisje & $\begin{array}{l}\text { mirë + qeverisje } \\
\text { good +governance }\end{array}$ & good governance & keqqeverisje & $\begin{array}{l}\text { keq + qeverisje } \\
\text { bad + governance }\end{array}$ & $\begin{array}{l}\text { bad } \\
\text { governance }\end{array}$ \\
\hline
\end{tabular}

The antonymy between the meanings of compound words of adjectives is more common in the Albanian language; antonymic adjectives of the type:

noun + noun: dëmprurës (maleficent) - dobiprurës (beneficent); fitimprurës (profitable) - jofitimprurës (unprofitable); etc. and the type:

noun + adjective: dorëlirë (spendthrift) — dorështrënguar (tightwad); vullnetfortë (strong-willed) — vullnetdobët (weakwilled); zemërmirë (kindhearted) — zemërkeq (evil-hearted), etc.

Most of these examples of antonymic adjectives are possessive compounds; therefore in those pairs the meanings are opposed which essentially indicate the characteristics of people ${ }^{1}$.

In the following, antonymic noun pairs are presented which are used in the economic terminology: akordim (accordance) çakordim (discordance); analizë (analysis) - sintezë (synthesis); asimetri (asymmetry) - simetri (symmetry); barazi (equality) — pabarazi (inequality); blerje (purchase) — shitje (sale); blokim (blockage) — zhblokim (unblocking); centralizim (centralization) - decentralizim (decentralization); dëmtim (damage) - zhdëmtim (compensation); doganim (imposition of customs duties) - zhdoganim (payment of customs duties); kamje (wealthiness, wealth) - skamje (poorness, poverty); kredi (credit) - debi (debit); ligjshmëri (legality) — paligjshmëri (illegality); makrostrukturë (macrostructure) mikrostrukturë (microstructure), marrës (receiver, donee) - dhënës (donor); mbingarkesë (overload) - nënngarkesë (underload); ngarkimi (loading) - shkarkimi (unloading); normë (norm) - jonormë (non-norm); pagim (payment) - mospagim (non-payment); punësim (employment) - papunësi (unemployment); rregullim (regulation) - çrregullim (deregulation); saktësi (accuracy) - pasaktësi (inaccuracy); shitës (seller) - blerës (buyer); shtrenjtësi (expensiveness) - lirësi (cheapness); zbatim (implementation) - moszbatimi (non-implementation) etc.

${ }^{1}$ Samara, M.: “Çështje të antonimisë në gjuhën shqipe”, ASHSH IGJL, Tiranë, 1985, f. 153. 


\section{Antonymic adjective pairs which are used in the economic terminology:}

afatshkurtër (short-term) - afatgjatë (long-term); aktiv/e (active) - joaktiv/e (inactive); i/e balancuar (balanced) - i/e pabalancuar (unbalanced); i/e barabartë (equal) - i/e pabarabartë (unequal); detyrues/e (binding) - jodetyrues/e (nonbinding); i/e drejtë (fair) - i/e padrejtë (unfair); ekonomik/e (economical) - joekonomik/e (uneconomical); i/e fortë (strong) - i/e dobët (weak); i/e hapur (open) - i/e mbyllur (closed); kapitalist/e (capitalist) - jokapitalist/e (non-capitalist); ligjor/e (legal) - kundërligjor/e (illegal); miqësor/e (friendly) - armiqësor/e (hostile); i/e miratuar (approved) - i/e refuzuar (rejected); i/e ngarkuar (loaded) - i/e shkarkuar (unloaded); optimist/e (optimistic) - pessimist/e (pessimistic); i/e pasur (rich) - i/e varfër (poor); i/e përdorur (used) — i/e papërdorur (unused); i/e pranueshëm (acceptable) - i/e papranueshëm (unacceptable); prodhues/e (productive) - joprodhues/e (unproductive); i/e qëndrueshëm (stable) - i/e paqëndrueshëm (unstable); real/e (real) - joreal/e (unreal); i ri/e re (junior) - i/e vjetër (senior); i/e rregullt (regular) - i/e parregullt (irregular); i/e saktë (accurate) - i/e pasaktë (inaccurate); i/e sigurt (safe) - i/e pasigurt (unsafe); i/e vlershëm (valid) - i/e pavlershëm (invalid); i/e zhvilluar (developed) - i/e pazhvilluar (undeveloped).

\section{Conclusion}

The antonymy as a linguistic phenomenon has been known since ancient times and in many points still arguable.

Based on practical and theoretical studies, we can encounter errors when trying to place a boundary between complete, partial, contextual, and intermediate antonyms. Nouns and verbs can form complete antonyms, while this is less common with adjectives and adverbs.

The antonyms of lexical-semantic affiliation are mainly nouns and qualitative adjectives; antonymic noun pairs: ekuilibër (balance) - desekuilibër (imbalance); aktiv (i bilancit) (active balance) - pasiv (i bilancit) (debit balance); debi (debit) kredi (credit); deficit (deficit) - suficit (surplus) etc.

\section{In the category of nouns, the verbal nouns differ:}

të ardhura (income) — të shpenzuara (expenses); shitje (sale) — blerje (purchase); importim (import) — eksportim (export); bllokim (blocking) - zhbllokim ( i fondeve) (unblocking) (of funds) etc.

Antonymic qualitative adjective pairs: (kontratë) afatgjatë (long-term) (contract)) - (kontratë) afatshkurtër (short-term (contract)); (kapital) i ndryshueshëm (variable) (capital)) —(kapital) i pandryshueshëm (invariable) (capital)); (fitim) $i$ shpërndarë (distributed (profit)) - (fitim) i pashpërndarë (undistributed (profit)) etc.

The antonymy is not unknown to terminology, but on the contrary, in terminology, it becomes a means of expression of the indispensable and inevitable phenomena of science. (of expressing the indispensable and inevitable occurrences of science.) This is due to the fact that the term is also a word, and that to some extent it is subject to these processes, the result of which are the ambiguous terms, the synonymic terms, and the antonymic term pairs.

\section{References}

[1] Duro, A. (2009). "Termi dhe fjala në gjuhën shqipe" IGJL, Fan Noli, Tiranë.

[2] Fjalor $i$ termave të gjuhësisë shqip-rusisht- frëngjisht- anglisht-gjermanisht -italisht (1975), ASHSH, IGJL, Sektori i terminologjisë, Tiranë.

[3] Fjalor i termave të transportit shqip-anglisht-frëngjisht-rusisht (2002), ASHSH, IGJL, Tiranë.

[4] Gorani, H. (2009). "Problemet kuptimore në terminologjinë ekonomike" në Gjendja dhe zhvillimi i terminologjisë shqipe, probleme e detyra", Ashsh \& Ashak, Tiranë.

[5] Memushaj, R. (2004). "Hyrje në gjuhësi", Dituria, Tiranë. 
[6] Pllana, G.: "Formimi i terminologjive teknike mbi bazën e leksikut të përgjithshëm”, QSA, Tiranë, 2017.

[7] Pllana, S.: "Terminologjia bazë e mekanikës në gjuhët shqipe dhe angleze", QSA, Tiranë, 2014.

[8] Pllana, S.; Breznica-Pllana, A. (2018). "Disa çështje të formimit të antonimeve folje e ndajfolje në terminologjinë e ekonomisë në gjuhën shqipe", Seminari i gjuhës shqipe, USHT Tetovë.

[9] Pllana, S.; Çitaku, F.; Arifaj, S.; Pllana, G. (2011). "Problem of Antonyms in the Mechanics Technology in Albanian and English", Sut Scientific Observer, Academic and scientific journal of linguistics, literature, education and culture published annually by the department of English language and literature State University of Tetova, Vol. 1, Issues $2 \& 3$.

[10] Pllana, S.; Pllana, G. (2014). "About Some Manifestations of Antonymy and Synonymy in the Terminology of Economics", Mediterranean Journal of Social Sciences MCSER Publishing, Rome-Italy, Vol. 5, No. 13, June 2014.

[11] Rexha, N. (2009). "Fjalor i biznesit, ekonomikës dhe finances anglisht-shqip, shqip-anglisht", ASAU, Prishtinë.

[12] Sager, J. (1990). "A practical course in Terminology Processing", Amsterdam, Philadelphia: John Benjamins.

[13] Samara, M. (1985)."Çështje të antonimisë në gjuhën shqipe”, ASHSH, Tiranë.

[14] Samara, M. (1999). "Fjalor i antonimeve në gjuhën shqipe", ASHSH, IGJL, Tiranë.

[15] Thomaj, J. (2006). "Leksikologjia e gjuhës shqipe", Botimet TOENA, Tiranë. 